\title{
Ultralow-energy vibrational quenching in ionic collisions: Isotope effects in $\mathrm{Li}^{+}+\mathrm{D}_{2}$ encounters
}

\author{
S. Bovino, E. Bodo, and F. A. Gianturco* \\ Department of Chemistry and CNISM, University of Rome La Sapienza, Piazzale A. Moro 5, 00185 Rome, Italy
}

(Received 13 December 2007; published 18 April 2008)

\begin{abstract}
The collisional, superelastic encounters at ultralow energies of $\mathrm{Li}^{+}$with $\mathrm{D}_{2}$ are computed using the exact coupled-channel dynamics, and using an ab initio potential energy surface discussed in earlier work. The changes in the target rovibrational structure due to the isotopic substitution, and in its rovibrational wave functions, are seen to have a marked effect, under the collision conditions of vanishing relative energy, on the corresponding dynamical attributes, allowing one to make specific predictions on the possible use of isotopic variants in cold trap processes.
\end{abstract}

DOI: 10.1103/PhysRevA.77.042716

PACS number(s): 34.50.Ez, 34.50.Lf

\section{INTRODUCTION}

The achievements of Bose-Einstein condensation in 1995 in dilute gases of ${ }^{87} \mathrm{Rb}[1],{ }^{7} \mathrm{Li}$ [2], and ${ }^{23} \mathrm{Na}$ [3] have strongly shifted the focus of atomic physics research, thereby extending the above achievements to several other alkalimetal species, to the study of several properties of BoseEinstein condensates and to the quantum degeneracy in Fermi gases such as ${ }^{40} \mathrm{~K}$ [4]. Thus, cold quantum matter has proved to exhibit a new range of unexpected properties and therefore its extension to molecular systems has become increasingly more attractive and active (e.g., see Refs. [5-7] for recent reviews). The study of Bose-Einstein condensates and of the Fermi degeneracy in dilute gases typically requires temperatures between $1 \mathrm{nK}$ and $1 \mu \mathrm{K}$, although new quantum properties start to appear at temperatures around 1 $\mathrm{mK}$, when the de Broglie wavelength becomes large compared to atomic and molecular dimensions. Hence, when such conditions are reached, collisions become fully quantum mechanical and are primarily sensitive to the long-range interactions, i.e., more generally, to the shapes and features of the intermolecular potentials close to their dissociation limits and to their effects on the dynamics at vanishing energies. Thus, the region below $1 \mathrm{mK}$ is usually described as the ultracold regimes of the molecular systems.

Once molecular systems are involved, their internal degrees of freedom, and the interplay of them with the interaction potentials in the ultracold regime, become important and therefore research has recently shifted to the ability to manipulate molecules at such regimes and to the possible control of reaction processes by modifying initial state populations, especially when dealing with polar systems where interactions are of longer range than in the neutral atomic gases [8-10].

Since rotations and vibrations are naturally the molecular channels more accessible at ultralow energies, it has become of interest to try to establish through quantum calculations in order to guide possible experiments, the relative efficiency of energy redistributions during superelastic collisions where the initial internal states of the molecule are taken to be

\footnotetext{
*Corresponding author; fa.gianturco@caspur.it; FAX: +39.06 .49913305 .
}

controlled (i.e., preselected) during the partner preparations in the cold and ultracold traps.

We have recently analyzed such changes of relative quenching efficiency in neutral $[11]$ and ionic $[12,13]$ systems in order to uncover possible trends of behavior and the effects on specific scattering attributes which are modified when molecular internal states are changed.

In the present work, taking the stronger ionic interaction as an example, we further consider the dynamical consequences of "tuning" the masses of the target molecule by employing its isotopic variant during the scattering calculations and by examining the quenching efficiency changes induced by such a simple, physically feasible, control of the dynamical parameters.

There are various reasons why ionic partners in cold traps are acquiring relevance in the area of ultracold dynamics: The cooling of molecular ions in Pauli traps has led to the formation of Coulomb "crystals" and, in general, of translationally cold partners $[14,15]$ with the view of analyzing the cooling (collisional) behavior of such species with a variety of rare gas partners. Furthermore, the influence of isotopic substitution in such processes has been recently computationally analyzed and has led to the finding of unexpectedly large effects [16]. We therefore thought it worthwhile to pursue such an analysis for an alkali-containing system as the present one.

Section II will briefly outline the general features of the interaction potential and the quantum scattering equations, while the results of the present analysis will be given in Sec. III. Section IV will finally report our present conclusions.

\section{INTERACTION AND DYNAMICS}

In the present work we employ once more the adiabatic electronic ground state of the $\mathrm{Li}^{+}+\mathrm{H}_{2}$ system, replacing the $\mathrm{H}_{2}$ masses with those of $\mathrm{D}_{2}$. We shall deal with collision energies well below the barrier to the reaction [17]

$$
\mathrm{Li}^{+}+\mathrm{D}_{2} \rightleftharpoons \mathrm{LiD}^{+}+\mathrm{D}
$$

which requires at least $4.4 \mathrm{eV}$ of collision energy or the $\mathrm{D}_{2}$ target vibrationally excited above $v=14$. Starting with vibrationally "hot" $\mathrm{D}_{2}$ molecules up to the ninth level, and considering only vanishingly small collision energies, all encounters will be below the reaction channel thresholds and 

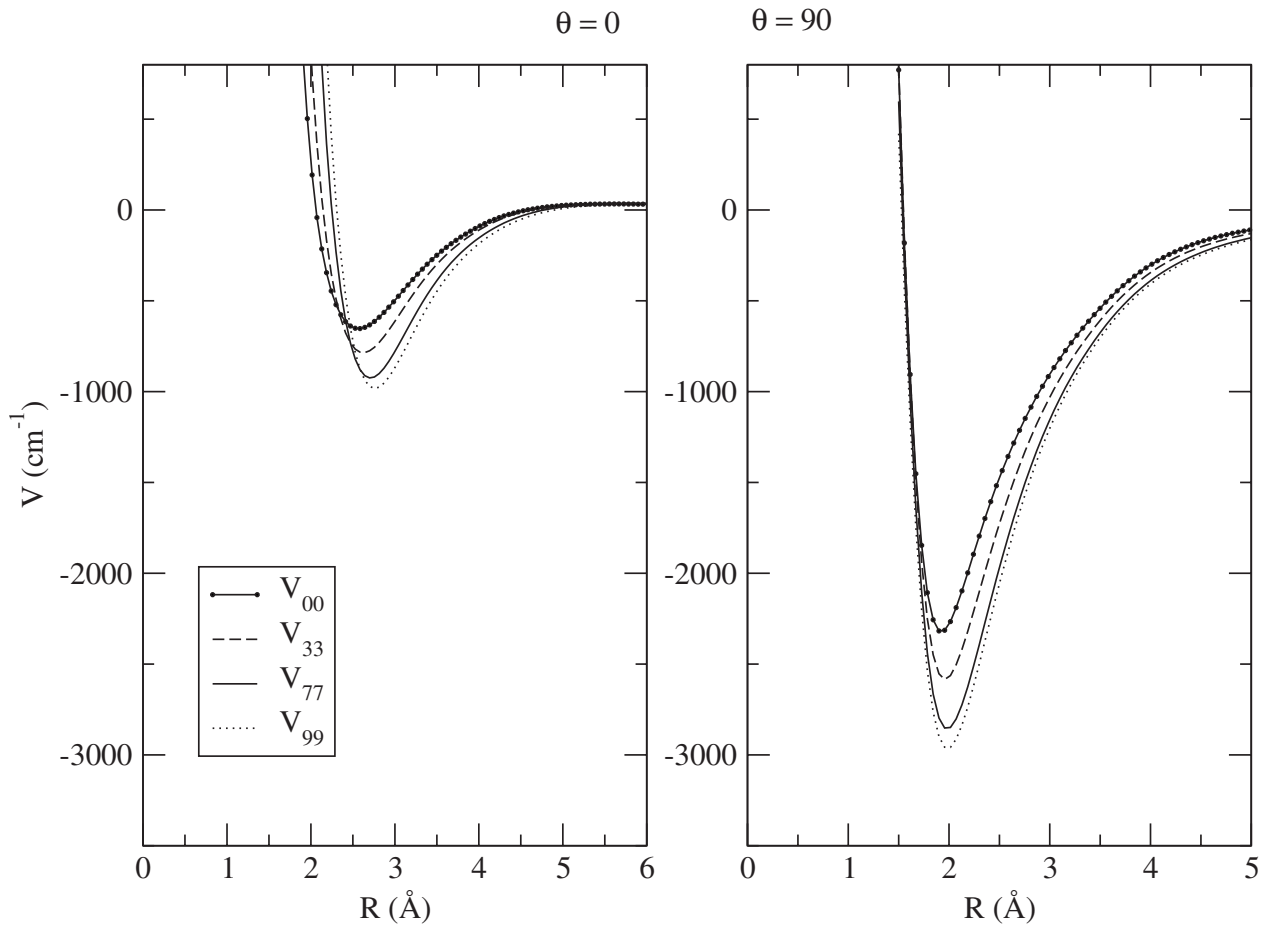

FIG. 1. Computed diagonal matrix elements for the vibrational coupling in the $\mathrm{Li}^{+}+\mathrm{D}_{2}(v)$ system. Two different orientations of the potential are considered. therefore only the inelastic processes (2) are open at the considered energies,

$$
\mathrm{Li}^{+}+\mathrm{D}_{2}(v \leq 9) \rightleftharpoons \mathrm{Li}^{+}+\mathrm{D}_{2}\left(v^{\prime}<v\right) .
$$

The details of the reactive surface have been discussed before $[17,18]$ and therefore will not be repeated here. Suffice it to say that the ionic system interaction at long range is dominated by the charge-quadrupole and the dipole-polarizability contributions coming from the $\mathrm{D}_{2}$ target.

Since we are chiefly interested in assessing the isotopic effects on the vibrational quenching process, we obtained the vibrational wave functions and vibrational levels of $\mathrm{D}_{2}$ by numerically solving the diatomic Schrödinger equation using the potential of Ref. [19]. We then obtained the individual coupling matrix elements between asymptotic states by numerical convolution over the $\mathrm{Li}^{+}+\mathrm{D}_{2}$ interaction potential mentioned above $[17,18]$,

$$
V_{v v^{\prime}}(R, \vartheta)=\left\langle\varphi_{v}|V(R, \vartheta, r)| \varphi_{v^{\prime}}\right\rangle
$$

which gives us a measure of the coupling strength induced by the interaction in driving the $v \rightarrow v^{\prime}$ process during the collision.

An example of the behavior of the diagonal elements of the coupling matrix originating from the integrals of Eq. (3) is shown in the two panels of Fig. 1, where two different values of $\vartheta$, the orientational Jacobi angle, are considered: The collinear approach on the left-hand panel and the $\mathrm{C}_{2 v}$ approach on the right-hand panel.

One clearly sees that, as $v$ increases, the potential wells move out to larger distances from the target molecule and the coupling strength also increases as one moves to initial states with higher $|v\rangle$ labeling: A result also found, naturally enough, in the case of the $\mathrm{H}_{2}$ partner studied before [13].
The quantum dynamics was treated within a timeindependent formulation, using the familiar coupled-channel (CC) approach in a space-fixed reference frame and employing our modified variable phase integrator to handle strongly, coupled, long-range potentials as in ionic reactions. The $\mathrm{CC}$ method is well known and has been given many times before $[12,13,20]$. The computed $\mathbf{K}$ matrix allows to obtain the scattering $\mathbf{S}$ matrix and the corresponding state-to-state superelastic cross sections [20].

When, at vanishing collision energies, we reach the $s$-wave dominated scattering regime we can then expand the elastic S-matrix elements in powers of $k[6,21]$,

$$
S_{v j, v j} \simeq 1+2 i \delta_{v j}(k)=1-2 i k\left(\alpha_{v j}-\beta_{v j}\right)=1-2 i k a_{v j},
$$

which allows us to obtain the complex scattering length, $a_{v j}$, for the $|v j\rangle$ channel and its real, $\alpha_{v j}$, and imaginary part, $\beta_{v j}$. They help us, in turn, to define the elastic and inelastic components of the partial integral cross sections at the vanishing energy regimes

$$
\begin{aligned}
& \sigma_{v j, v j}^{\mathrm{el}} \simeq 4 \pi\left|a_{v j}\right|^{2} \quad \text { as } k_{v j} \rightarrow 0, \\
& \sigma_{v j, v j}^{\text {inel }} \simeq 4 \pi \beta_{v j} k_{v j} \quad \text { as } k_{v j} \rightarrow 0 .
\end{aligned}
$$

We further obtain the quenching rates down to all open channels for any selected initial state $|v j\rangle$,

$$
R_{v j}=\frac{4 \pi \hbar}{\mu} \beta_{v j}
$$

The size and sign of the complex scattering length for each initial channel $|v j\rangle$ gives us information on the behavior of the interaction potential at vanishing collision energies close to the dissociation limit of the ionic complex [22], i.e., we obtain data on the existence of either zero-energy resonances (virtual states) or of complex bound states located very near 


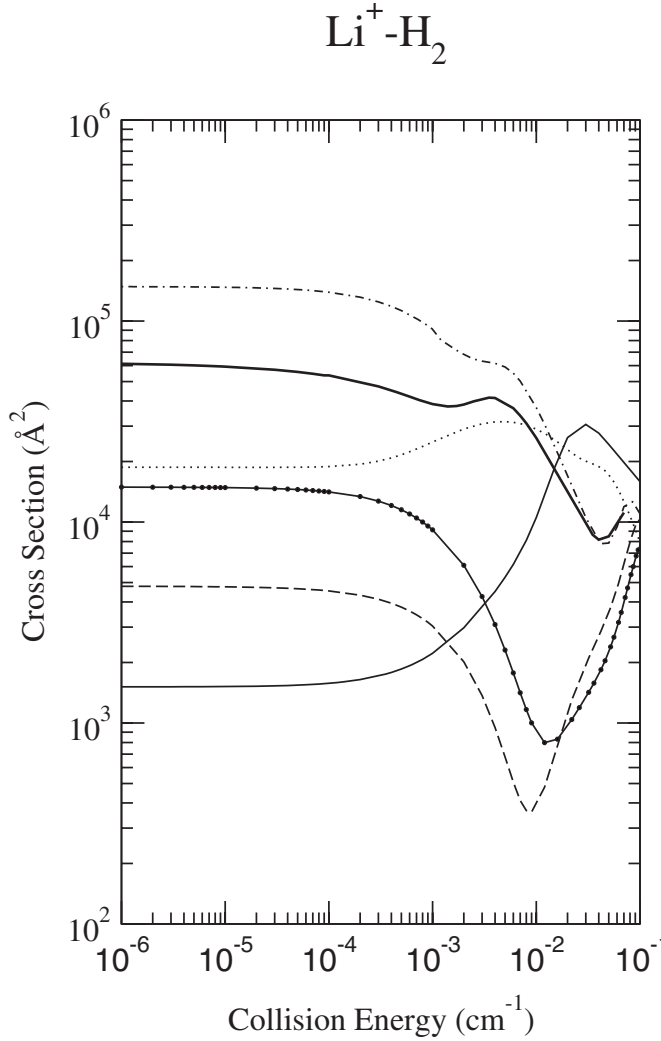

$\mathrm{Li}^{+}-\mathrm{H}_{2}$
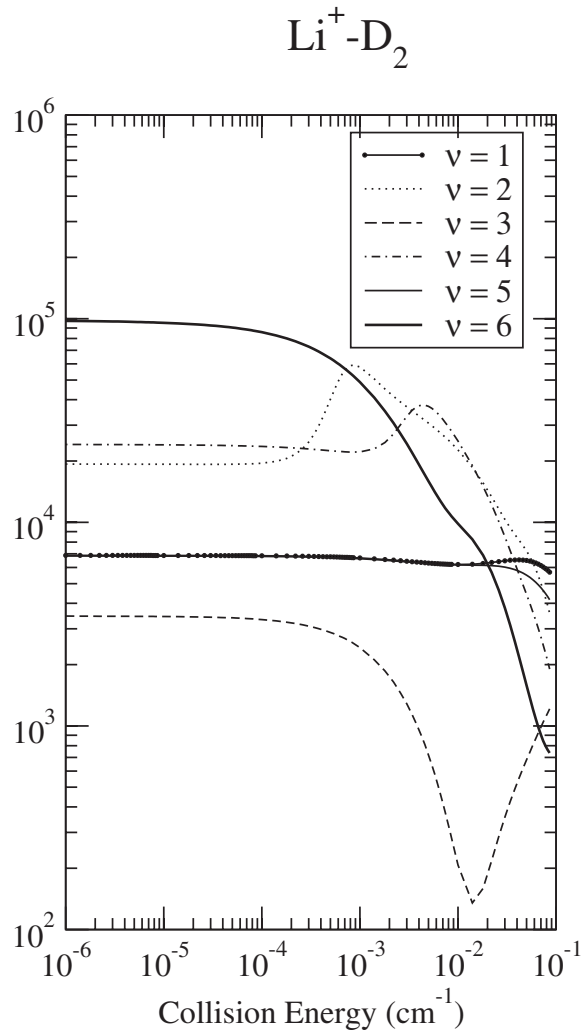

FIG. 2. Vibrationally elastic cross sections for the two isotopic variants of the hydrogen molecule and for different initial vibrational states of the partners. to the dissociative threshold by inspecting the presence of either negative or positive values of the real part of the corresponding scattering length [22].

The complex energies associated with such states are further given as [21,22]

$$
\begin{aligned}
E & =-\frac{\hbar^{2}}{2 \mu\left|a_{v j}\right|^{2}}\left(\cos 2 \gamma_{v j}+i \sin 2 \gamma_{v j}\right) \\
& =E_{v j}-\frac{i}{2} \Gamma_{v j},
\end{aligned}
$$

where $\gamma_{v j}=\tan ^{-1} \beta_{v j} / \alpha_{v j}$. We can now use the present calculations to assess the effect of isotopic substitution on the scattering attributes of this ionic system at vanishing collision energies and to see how such mass changes can be amplified by the corresponding subtle changes of its interaction forces. In the area of ultracold dynamics, in fact, a recent experimental study on the atomic photoassociation of rubidium atoms [23] has underlined the marked efficiency change observed when going from the formation of ${ }^{85} \mathrm{Rb}_{2}$ to ${ }^{87} \mathrm{Rb}_{2}$ molecules and has attributed such changes to modifications in the resonant coupling strength when going from one reduced mass to the other. The present analysis, albeit not yet supported by experimental evidence, aims indeed at showing the effect of isotopic modifications on molecular energy redistribution processes at ultralow collision energies in the case of possible candidates for cold Pauli traps leading to the formation of Coulomb "crystals" [14,15].

\section{RESULTS AND DISCUSSION}

As mentioned before, the dynamics at vanishing energies has revealed in previous studies (e.g., see Refs. [16,23-26]) that the isotopic substitution of one of the partners can have marked consequences on the collisional attributes, exhibiting important changes in their behavior. The present study is therefore a further computational example, on an ionic system, of how such isotopic changes are reflected on the vibrational quenching reactions involving ionic interactions.

An initial comparison of changes induced by the isotopic substitution can be seen in the two panels reported by Fig. 2: We show the behavior of the elastic cross sections down to the Wigner's regime [27], where the latter quantities become constant with energy.

We clearly see, in general terms, that all cross sections for the $\mathrm{D}_{2}$ target span a narrower energy range at vanishing energies (a little more than one order of magnitude) than is the case for $\mathrm{H}_{2}$ (where two orders of magnitude are spanned). Furthermore, the size ordering changes a great deal upon isotopic substitution: The largest cross section comes from the $v=4$ initial level for $\mathrm{H}_{2}$, while it is from $v=6$ for $\mathrm{D}_{2}$. Additionally, we see that, when going from $\mathrm{H}_{2}$ to $\mathrm{D}_{2}$ as a target, the presence of possible Ramsauer-Townsend (RT) minima in the cross sections [28] is moving to higher energies and for different initial states.

The differences in the cross-section behavior when performing isotopic replacement are even more evident when we look at the inelastic cross sections associated with vibrational quenching processes from different initial levels but 


$$
\mathrm{Li}^{+}-\mathrm{H}_{2}
$$

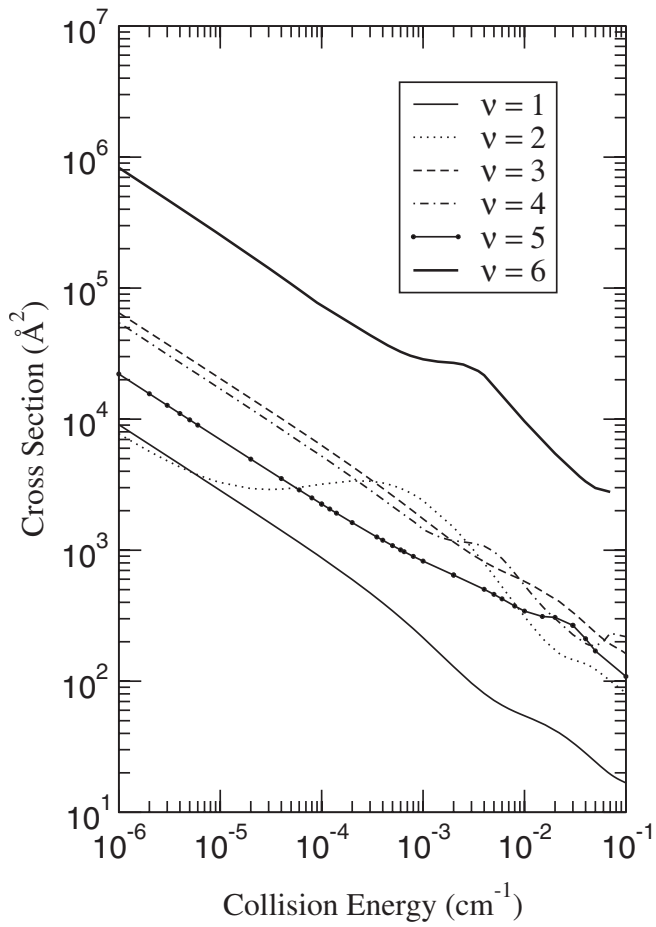

$\mathrm{Li}^{+}-\mathrm{D}_{2}$

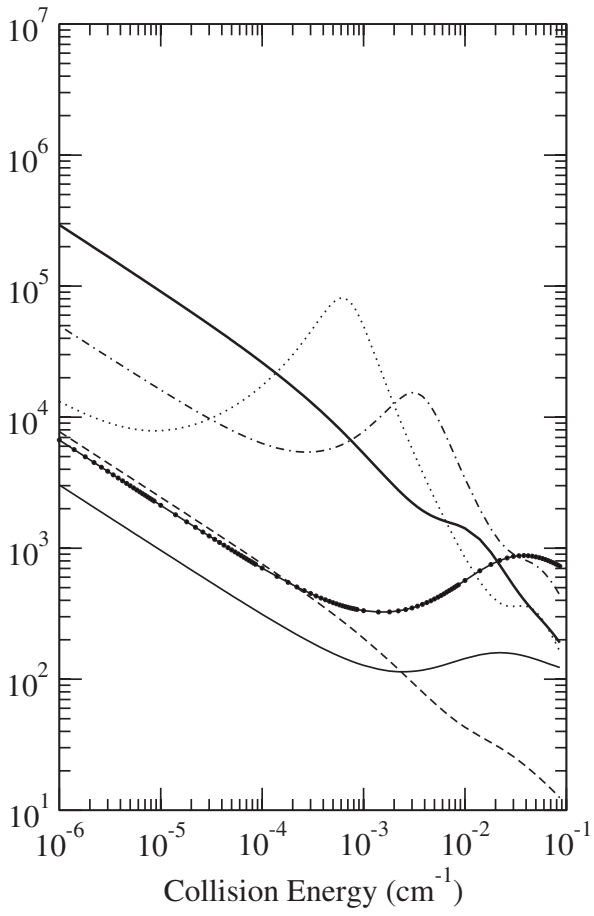

FIG. 3. Computed inelastic cross sections from different initial vibrational levels and down to all lower-lying rotovibrational states. Left-hand panel, $\mathrm{H}_{2}$ target; right-hand panel, $\mathrm{D}_{2}$ molecule. summed over all final rotovibrational levels. The calculations are reported by the two panels of Fig. 3 .

The results show that the inelastic cross section is nearly always reduced upon replacing $\mathrm{H}_{2}$ with $\mathrm{D}_{2}$ : The inelastic processes span about two orders of magnitude in both cases and the larger cross sections occur when $\mathrm{H}_{2}$ is the molecular partner. Furthermore, we see that the relative ordering sizes change when going from $\mathrm{H}_{2}$ to $\mathrm{D}_{2}$ and two prominent quenching maxima appear in the cross sections for the latter target, while missing for the $\mathrm{Li}^{+}+\mathrm{H}_{2}$ case.

The closer spacing's between bound states exhibited by $\mathrm{D}_{2}$ versus $\mathrm{H}_{2}$ may be responsible for a more efficient dynamical coupling during collisions in the former case, although the scaling of the reduced mass causes also a reduc-
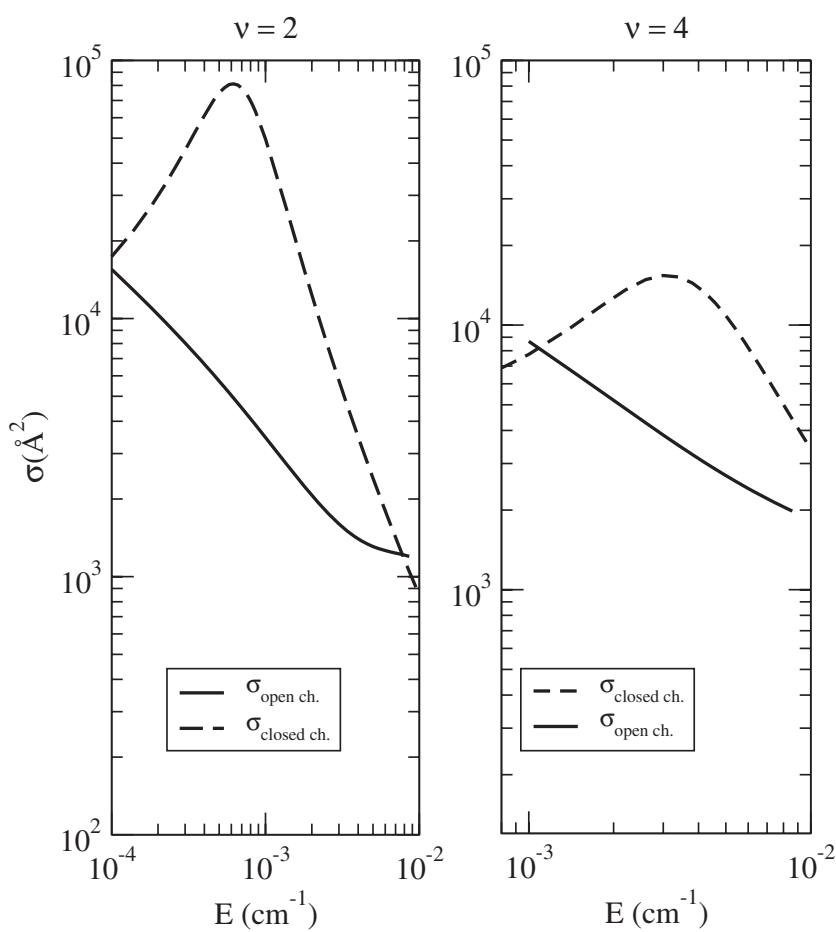

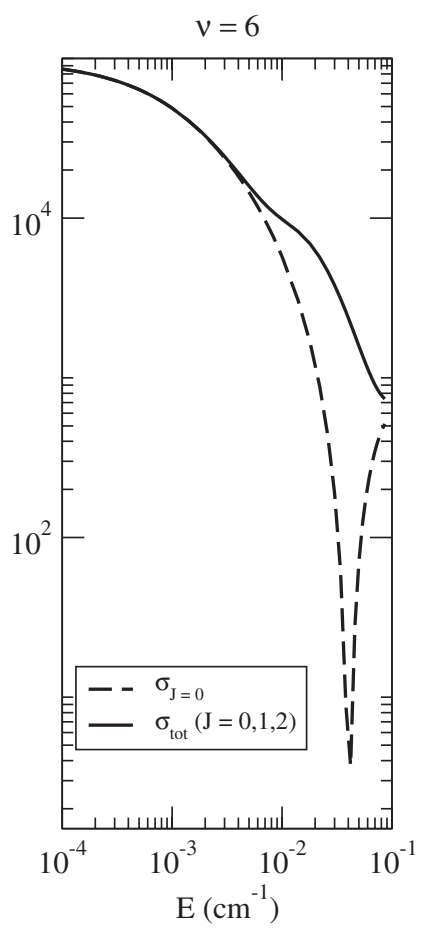

FIG. 4. Computational tests on the inelastic cross section peaks shown by Fig. 3, right-hand panel. The $v=2$ and $v=4$ cases are reported in the left-hand and central panels. The right-hand panel shows the elastic cross section for $v=6$. See text for details. 
TABLE I. Real $(\alpha)$ and imaginary $(\beta)$ components of the scattering lengths, energy locations $(E)$, and lifetimes $(\Gamma)$ of the virtual and bound states, and quenching rates $\left(R_{\nu}\right)$.

\begin{tabular}{lcccccc}
\hline \hline$\nu$ & $\alpha(\AA)$ & $\beta(\AA)$ & $E\left(\mathrm{~cm}^{-1}\right)$ & $\Gamma_{\nu}(\mathrm{ns})$ & $R_{\nu}^{\mathrm{D}_{2}}(T \rightarrow 0)$ & $R_{\nu}^{\mathrm{H}_{2}}(T \rightarrow 0)$ \\
\hline 1 & 23.39 & $9.41 \times 10^{-2}$ & $-1.20 \times 10^{-2}$ & 27.3 & $2.93 \times 10^{-12}$ & $1.12 \times 10^{-11}$ \\
2 & 39.19 & $40.9 \times 10^{-2}$ & $-4.28 \times 10^{-3}$ & 29.7 & $1.27 \times 10^{-11}$ & $9.17 \times 10^{-12}$ \\
3 & 16.59 & $24.0 \times 10^{-2}$ & $-2.38 \times 10^{-2}$ & 3.8 & $7.49 \times 10^{-12}$ & $8.42 \times 10^{-11}$ \\
4 & 43.77 & $15.4 \times 10^{-1}$ & $-3.42 \times 10^{-3}$ & 10.9 & $4.82 \times 10^{-11}$ & $6.69 \times 10^{-11}$ \\
5 & 23.31 & $20.7 \times 10^{-2}$ & $-1.21 \times 10^{-2}$ & 12.3 & $6.46 \times 10^{-12}$ & $4.14 \times 10^{-11}$ \\
6 & -87.76 & $91.0 \times 10^{-1}$ & $-8.29 \times 10^{-4}$ & 15.2 & $2.83 \times 10^{-10}$ & $1.19 \times 10^{-9}$ \\
7 & 29.51 & $21.7 \times 10^{-1}$ & $-7.42 \times 10^{-3}$ & 2.4 & $6.77 \times 10^{-11}$ & $1.01 \times 10^{-10}$ \\
8 & 24.93 & $12.8 \times 10^{-1}$ & $-1.05 \times 10^{-2}$ & 2.4 & $3.99 \times 10^{-11}$ & $6.43 \times 10^{-10}$ \\
9 & 32.86 & 10.54 & $-4.48 \times 10^{-3}$ & 0.8 & $3.28 \times 10^{-10}$ & $6.16 \times 10^{-10}$ \\
\hline \hline
\end{tabular}

tion of the inelastic flux. Thus, it is not obvious to understand the nature of the resonances on the right-hand panel of Fig. 3 unless further calculations are carried out. A sampling of further checks on the scattering attributes is therefore reported in the three panels of Fig. 4.

In the case of the two quenching maxima seen for $v=2$ and 4 around $E_{\text {coll }} \sim 10^{-3} \mathrm{~cm}^{-1}$, the calculations in the lefthand and central panels report them for two different situations: The dashed curves repeat the results of Fig. 3, where three closed vibrational channels were included for each quenching process, while the solid lines show the same calculations but this time without vibrational closed channels. The ensuing suppression of the quenching cross sections in the latter computations is indicative of the fact that the enhancements are due to the presence of Feshbach resonances, with virtual excitations into the vibrational closed channels occurring for the isotopic species.

The data in the right-hand panel, on the other hand, report the elastic cross section from the $v=6$ level and contain the three dominant components of the total angular momentum involved in that collision. We clearly see there that the $s$-wave scattering generates a RT-type minimum in its partial cross section, while the additional contributions mask such an effect in the total cross sections. The additional minimum in the elastic cross section for $v=3$, the only other minimum feature shown by the $\mathrm{D}_{2}+\mathrm{Li}^{+}$case, has also been analyzed (not shown here) and found to be related to different contributing partial waves which interfere at that energy by producing a dip in the elastic cross sections, without however being caused by a virtual state.

The results gathered in Table I further summarize the behavior of the scattering attributes in the case of the $\mathrm{D}_{2}$ molecular partner, as a function of the initial vibrational level from which the quenching is considered. A pictorial representation of the behavior of the vibrational quenching rates reported in Table I is also given by Fig. 5, where their dependence on the initial vibrational level is compared for the $\mathrm{H}_{2}$ and $\mathrm{D}_{2}$ molecular partners.

In a recent study [28] reporting elastic and inelastic processes for $\mathrm{Li}+\mathrm{Li}_{2}(v, j)$ collisions, it was computationally shown that the isotopic variant with ${ }^{7} \mathrm{Li}$ exhibited smaller inelasticity than that produced by the system containing ${ }^{6} \mathrm{Li}$ isotopes. Our earlier calculations [11] on the $\mathrm{Li}_{2}+\mathrm{He}$ and $\mathrm{H}_{2}+\mathrm{H}$ systems had further shown that treating the isotopic masses as disposable parameters provided a dramatic reduc-

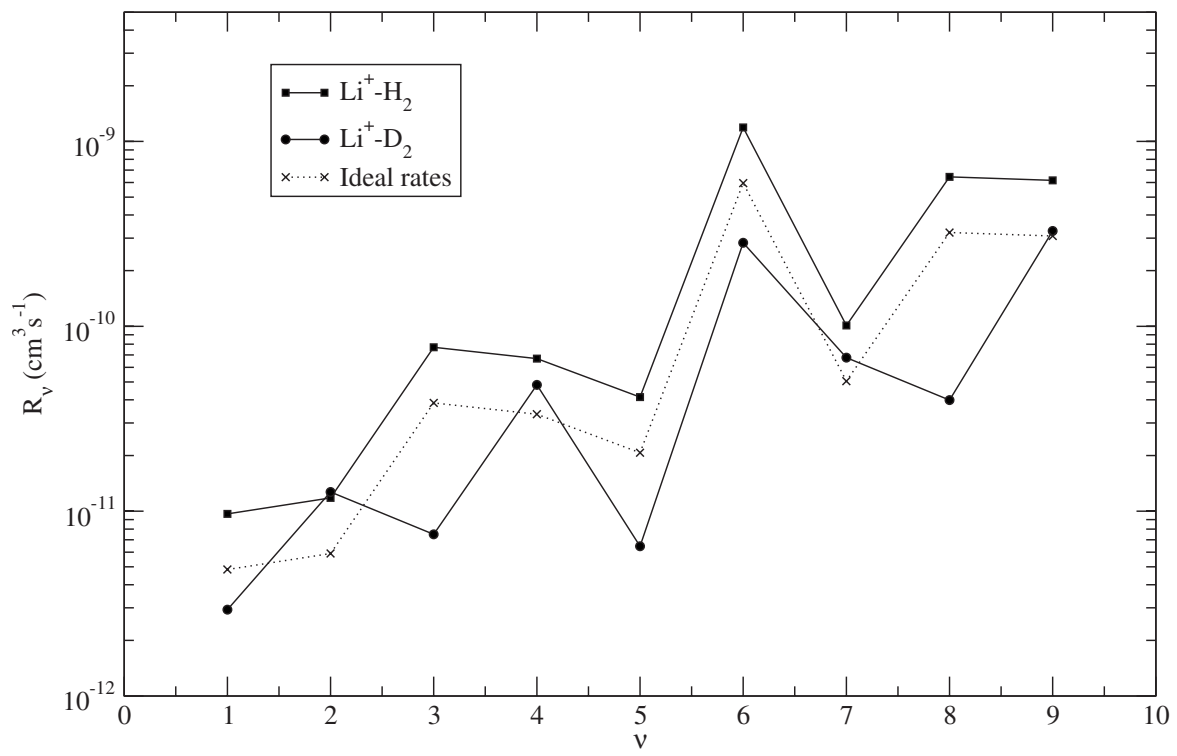

FIG. 5. Computed vibrational quenching rates for $\mathrm{Li}^{+}$colliding with $\mathrm{H}_{2}$ (filled squares) and with $\mathrm{D}_{2}$ (filled circles). See text for details and for the definition of "ideal rates." 
tion of the quenching dependence on the initial vibrational level of the heavier molecular partner.

The results of Fig. 5 indeed follow the trends of these previous studies and indicate that, over the whole range of vibrational values examined, the heavier molecular partner yields quenching rates which are consistently smaller than in the case of the lighter molecular species and the differences are strongly dependent on the selected vibrational initial state of the target. Thus, the present results confirm that to introduce changes in the rotovibrational structure of the molecular partner has marked effects on the quenching efficiency of trap processes. To further prove this point, the dotted curve in the figure reports what we have labeled as "ideal rates," i.e., the rate values obtained from Eq. (7) by assuming the same $\beta$ 's as in the $\mathrm{Li}^{+}+\mathrm{H}_{2}$ case and only changing the reduced mass to that of the $\mathrm{D}_{2}$ target. We clearly see that the reduction effects on rates are not only due to mass changes but come from modifications appearing in the coupling dynamics upon isotopic substitution. Thus, we can suggest that, at such vanishing energies, the ionic interactions can cause substantial effects on the scattering observables when "tuning" the relevant reduced masses by isotopic replacements.

Another interesting element of information is given by the behavior of the real and imaginary parts of the computed scattering length as a function of the initial vibrational level: The actual numerical values are reported in Table I.

It is interesting to note here that for only one of the configurations examined in the calculations [that with $\mathrm{D}_{2}$ $(v=6)$ as initial state] we found a negative and large value of $\alpha_{v j}$. It corresponds to the presence of an RT minimum feature masked in Fig. 2 by the contributions beyond the dominant $s$-wave component. The $\mathrm{H}_{2}$ partner, on the other hand, had shown [13] three different arrangements which exhibited virtual state effects very close to the vanishing energy thresholds.

Thus, as the mass increases those states move away from threshold (apart from the $v=6$ configuration) and give rise to "proper" bound states located on the first Riemann's sheet [22] and further down in energy. Therefore, they have a reduced effect on the quenching efficiency.

In Table I we further report the lifetimes of the metastable bound states of the complex found in our calculations. We see there that all such states are rather long lived and their break-up efficiency increases with increasing $v$, with the exception again of the $v=6$ complex where a virtual state affects the behavior of the scattering attributes.

\section{PRESENT CONCLUSIONS}

In this work we have analyzed in some detail the consequences, on the vibrational quenching efficiency in an ionic trap, of replacing the molecular partner $\mathrm{H}_{2}$ with its isotopic variant $\mathrm{D}_{2}$. The dynamics was that of an ionic system where
$\mathrm{Li}^{+}$was the atomic partner and the collisional energy range considered was that of the ultralow energies.

The potential energy surface employed came from earlier $a b$ initio calculations carried out in our group [18] and the quantum dynamics was performed within the coupledchannel approach, using the rotovibrational basis of the $\mathrm{D}_{2}$ asymptotic target.

The computations reveal a clear effect on scattering observables from isotopic replacement, in keeping with the earlier findings with other systems at the ultralow-energy regimes $[11,16,24,26,28]$ and relate such changes to the induced potential modifications which, at such ultralow energies, affect the interaction close to the dissociative limit: The strong presence of virtual-state enhancement seen for the $\mathrm{Li}^{+}+\mathrm{H}_{2}$ case $[12,13]$ is here replaced by bound state formations further away from the energy threshold and therefore less effective in enhancing dynamical couplings between bound and continuum states at vanishing energies. As a consequence, we see that the individual inelastic cross sections are smaller (see Fig. 3) and the final quenching rates are consistently smaller for the $\mathrm{D}_{2}$ partner than for the $\mathrm{H}_{2}$ partner, the result being however dependent on the chosen initial vibrational state: Each selected vibrational energy content of the isotopic partner, in fact, induces different modifications of the scattering attributes at vanishing collision energies, both in the elastic and inelastic channels, thereby affecting by different amounts the final quenching rates.

The possible appearance of Ramsauer-Townsend minima in the elastic cross sections, an indication of virtual state existence at vanishing energies, is therefore not seen in the heavier system with respect to the lighter partner, where several of such minima were detected [13].

The closer spacing's between target vibrational states also increases here the importance of Feshbach resonances, features which are connected with improved couplings between asymptotically closed vibrational channels. Thus, the quantum calculations show that marked maxima in the inelastic cross sections (see Fig. 3) disappear when the coupling to closed asymptotic channels is switched off.

In conclusion, the present computational study indicates that, at least for strongly interacting ionic systems, the isotopic changes can be used as "tuning knobs" for experimentally controlling the vibrational quenching efficiency since the induced changes on the interaction, and on the partners' internal structures, have marked consequences on the elastic and inelastic cross sections at ultralow collision energies and could be amenable to observation in cold Pauli trap experiments $[14,15]$.

\section{ACKNOWLEDGMENTS}

The financial support of the University of Rome I Research Committee, of the CASPUR Supercomputing Consortium and of the MIUR PRIN project is gratefully acknowledged. 
[1] M. H. Anderson, J. R. Ensher, M. R. Matthews, C. E. Wieman, and E. A. Cornell, Science 269, 198 (1995).

[2] C. C. Bradley, C. A. Sackett, J. J. Tollett, and R. G. Hulet, Phys. Rev. Lett. 75, 1687 (1995).

[3] K. B. Davis, M. O. Mewes, M. R. Andrews, N. J. van Druten, D. S. Durfee, D. M. Kurn, and W. Ketterle, Phys. Rev. Lett. 75, 3969 (1995).

[4] D. De Marco and D. S. Jin, Science 285, 1703 (1999).

[5] E. Bodo and F. A. Gianturco, Eur. Phys. J. D 31, 423 (2004).

[6] E. Bodo and F. A. Gianturco, Int. Rev. Phys. Chem. 25, 313 (2006).

[7] J. M. Hutson and P. Soldán, Int. Rev. Phys. Chem. 25, 497 (2006).

[8] D. De Mille, Phys. Rev. Lett. 88, 067901 (2002).

[9] H. L. Bethlem and G. Meijer, Int. Rev. Phys. Chem. 22, 73 (2003).

[10] S. Chu, Rev. Mod. Phys. 70, 685 (1998).

[11] E. Bodo, F. A. Gianturco, and E. Yurtsever, Phys. Rev. A 73, 052715 (2006).

[12] E. Bodo and F. A. Gianturco, Europhys. Lett. 77, 33001 (2007).

[13] S. Bovino, E. Bodo, and F. A. Gianturco, J. Chem. Phys. 127, 224303 (2007).

[14] P. Bowe, L. Hornekaer, C. Brodersen, M. Drewsen, J. S. Hangst, and J. P. Schiffer, Phys. Rev. Lett. 82, 2071 (1999).
[15] K. Mølhave and M. Drewsen, Phys. Rev. A 62, 011401(R) (2000).

[16] G. Guillon, T. Stoecklin, and A. Voronin, Phys. Rev. A 75, 052722 (2007).

[17] R. Martinazzo, E. Bodo, F. A. Gianturco, and M. Raimondi, Chem. Phys. 287, 335 (2003).

[18] E. Bodo, F. A. Gianturco, and R. Martinazzo, Chem. Phys. 271, 309 (2001).

[19] W. Kolos and L. Walniewicz, J. Chem. Phys. 43, 2429 (1965).

[20] R. Martinazzo, E. Bodo, and F. A. Gianturco, Comput. Phys. Commun. 151, 187 (2003).

[21] N. Balakrishnan, R. Forrey, and A. Dalgarno, Chem. Phys. Lett. 280, 1 (1997).

[22] J. R. Taylor, Scattering Theory (Wiley, New York, 1972).

[23] A. Fioretti, O. Dulieu, and C. Gabbanini, J. Phys. B 40, 3283 (2007).

[24] T. Stoecklin and A. Voronin, Phys. Rev. A 72, 042714 (2005).

[25] E. Bodo and F. A. Gianturco, J. Phys. Chem. A 107, 7328 (2003).

[26] E. Bodo, F. A. Gianturco, and A. Dalgarno, J. Phys. B 35, 2391 (2002).

[27] P. E. Wigner, Phys. Rev. 73, 1002 (1948).

[28] G. Quéméner, J. M. Launay, and P. Honvault, Phys. Rev. A 75, 050701(R) (2007). 